\title{
QUÍMICA E COLORIMETRIA DA MADEIRA DE Eucalyptus grandis W. Mill ex Maiden TERMORRETIFICADA ${ }^{1}$
}

\author{
Antônio José Vinha Zanuncio², João Rodrigo Coimbra Nobre ${ }^{3}$, Javan Pereira Motta ${ }^{4}$ e Paulo Fernando \\ Trugilho $^{5}$
}

\begin{abstract}
RESUMO - A termorretificação visa melhorar as características para agregar valor à madeira através da aplicação de calor. O objetivo foi avaliar as alterações químicas e colorimétricas da madeira termorretificada de Eucalyptus grandis submetida a 170, 200 e $230^{\circ} \mathrm{C}$ por 3, 5 e 7 h. Os teores de extrativos, lignina, holocelulose, composição elementar e os parâmetros colorimétricos foram determinados. Os teores de extrativos aumentaram nos tratamentos a $170{ }^{\circ} \mathrm{C}$ e diminuíram a partir de $200{ }^{\circ} \mathrm{C}$, enquanto o teor de lignina total aumentou e o de holocelulose diminuiu a partir de $200^{\circ} \mathrm{C}$. O teor de carbono aumentou e o de oxigênio diminuiu a $230{ }^{\circ} \mathrm{C}$ por 5 e $7 \mathrm{~h}$. A termorretificação reduziu a claridade, matriz vermelho (a*) e matriz amarelo (b*) da madeira de eucalipto em todos os tratamentos.
\end{abstract}

Palavras-chave: Extrativo; Lignina; Tratamento térmico.

\section{CHEMICAL AND COLOR CHANGES IN HEAT TREATED Eucalyptus grandis W. Mill ex Maiden WOOD}

\begin{abstract}
The heat treatment aims to improve the features to add value to the wood through the application of heat. The objective of this work was to evaluate the chemical and colorimetric changes in Eucalyptus grandis wood heat treated at 170,200 and $230^{\circ} \mathrm{C}$ for three, five and seven hours. The content of extractives, lignin, holocellulose, elemental composition and colorimetric parameters were determined. The extractives content increased in treatments at $170^{\circ} \mathrm{C}$ and decreased from $200^{\circ} \mathrm{C}$, while total lignin increased and holocellulose decreased from $200^{\circ} \mathrm{C}$. The carbon content increased and oxygen content decreased at $230^{\circ} \mathrm{C}$ for five and seven hours. The heat treatment reduced lightness, $a^{*}$ coordinate (red coordinate) and $b^{*}$ coordinate (yellow coordinate) of eucalyptus wood in all treatments.
\end{abstract}

Keywords: Extractives; Lignin; Heat treatment.

\section{INTRODUÇÃO}

A área plantada com florestas de rápido crescimento no Brasil atingiu 6,5 milhões de hectares, com 74,8\% de espécies do gênero Eucalyptus (ABRAF, 2012). A madeira apresenta caráter higroscópico, suscetibilidade a agentes degradadores e coloração clara, o que dificulta seu uso (ABRUZZI et al., 2012; FURTADO et al., 2012; CARNEIRO et al., 2013; LIMA et al., 2013), tornando necessária a utilização de tratamentos adicionais, como a termorretificação, vaporização, acetilação etc.

O calor é um dos métodos mais difundidos para tratamento da madeira com temperatura variável, conforme a finalidade. A água da madeira é removida entre a temperatura ambiente e $100{ }^{\circ} \mathrm{C}$, as hemiceluloses apresentam alterações irreversíveis entre 100 e $250^{\circ} \mathrm{C}$,

\footnotetext{
${ }^{1}$ Recebido em 01.08.2013 aceito para publicação em 27.05.2014.

${ }^{2}$ Programa de Pós-Graduação em Ciência Florestal, Universidade Federal de Viçosa, UFV, Brasil. E-mail: <ajvzanuncio@yahoo.com.br>.

${ }^{3}$ Programa de Pós-Graduação em Ciência e Tecnologia da Madeira, Universidade Federal de Lavras, UFLA, Brasil. E-mail: <sokonobre@hotmail.com>

${ }^{4}$ Departamento de Tecnologia da Madeira, Universidade da Estado do Pará, Campus VIII - Marabá. E-mail:< javanmotta@uepa.br>

${ }^{5}$ Departamento de Ciências Florestais, Universidade Federal de Lavras, UFLA, Brasil. E-mail: <trugilho@dcf.ufla.br>.
} 
sendo totalmente destruídas entre 250 e $330^{\circ} \mathrm{C}$. A celulose é destruída entre 330 e $370^{\circ} \mathrm{C}$, e a $370^{\circ} \mathrm{C}$ ocorre degradação das ligninas (SCHAFFER, 1973).

A termorretificação consiste na aplicação de calor entre 160 e $230{ }^{\circ} \mathrm{C}$, dependendo das características do material desejado e da resistência da madeira (KOCAEFE et al., 2008; ESTEVES; PEREIRA, 2009). Este tratamento degrada as hemiceluloses, destruindo monômeros como arabinose, manose, galactose e xilose (BRITO et al., 2008; SEVERO et al.; 2012; BROSSE et al.; 2010), com liberação de extrativos como o ácido acético, furfural e mono, sesqui e diterpenos (ESTEVES et al., 2011). No entanto, as ligninas e celulose são menos afetadas (YILDIZ et al., 2006; TUMEN et al., 2010).

As alterações químicas melhoram a estabilidade dimensional, comportamento higroscópico e resistência biológica da madeira (ALMEIDA et al., 2009; BAL; BEKTAS, 2012; KORKUT, 2012; RATNASINGAM; IORAS, 2012), mas podem prejudicar suas propriedades mecânicas (SHI et al., 2007; GARCIA et al., 2012; DUNDAR et al., 2012; CADEMARTORI et al., 2012).

A degradação das hemiceluloses escurece a madeira termorretificada (ESTEVES et al., 2008), e reações químicas dos extrativos durante esse processo podem alterar as matrizes vermelho e amarelo (MOURA; BRITO, 2011).

O objetivo deste trabalho foi avaliar alterações químicas e colorimétricas na madeira de Eucalyptus grandis submetida à termorretificação.

\section{MATERIAL E MÉTODOS}

Três árvores de Eucalyptus grandis com 15 anos de idade foram utilizadas, por ser essa espécie uma das mais empregadas no Brasil e estar na idade recomendada para uso como madeira sólida. As árvores foram desdobradas e secas ao ar livre até a umidade de equilíbrio. As amostras foram, então, trituradas em moinho Willey e peneiradas. A fração retida entre as peneiras de 40 e 60 mesh foi dividida em amostras de $3 \mathrm{~g}$ absolutamente secas, submetidas à secagem a $100{ }^{\circ} \mathrm{C}$ e termorretificadas a 170,200 e $230^{\circ} \mathrm{C}$ por 3 , 5 e 7 h sob pressão atmosférica e na presença de ar.

O teor de extrativos totais da madeira de eucalipto foi determinado de acordo com a norma TAPPI 204 om-88; o de lignina insolúvel pelo método Klason modificado (GOMIDE; DEMUNER, 1986);o de lignina solúvel em ácido a partir do filtrado resultante da análise da lignina Klason pela leitura em espectrofotômetro (GOLDSCHIMID, 1971); e o de lignina total pela soma da lignina residual com a solúvel em ácido. As holoceluloses foram determinadas subtraindo os teores de lignina e extrativos de 100.

A análise elementar foi realizada conforme descrito por Paula et al. (2011). Os teores de carbono, hidrogênio e nitrogênio, em relação à massa seca de madeira, foram quantificados com um analisador universal da marca Elementar (modelo Vario MicroCube). O teor de oxigênio foi obtido subtraindo os teores de carbono, hidrogênio e nitrogênio de 100 .

A claridade (L), a coordenada vermelho-verde ou matiz vermelho (a*) e a coordenada azul-amarelo ou matiz amarelo ( $\mathrm{b}^{*}$ ) foram avaliadas de acordo com o sistema CIELAB 1976, conforme descrito por Pincelli et al. (2012), utilizando um espectrofotômetro Konica Minolta CM-2500D.

Todos os parâmetros avaliados foram realizados em triplicata e submetidos ao teste de Scott-Knott a $5 \%$ de probabilidade.

\section{RESULTADOS}

\subsection{Análise química}

O teor de extrativos variou entre 4,72 e 8,96\%, com menor valor nos tratamentos a $170{ }^{\circ} \mathrm{C}$ e maiores a $230{ }^{\circ} \mathrm{C}$ (Tabela 1$)$.

A lignina solúvel variou de 3,1 a 3,57\%, a insolúvel entre 28,76 e 37,18\% e as holoceluloses de 54,12 a 66,27\%. Os teores de lignina insolúvel e total aumentaram com o tratamento térmico, em detrimento das holoceluloses, mas o de lignina solúvel não foi afetado (Tabela 1).

\subsection{Análise elementar}

O teor de nitrogênio da madeira foi semelhante nas diferentes temperaturas, o de hidrogênio foi menor a partir de $200^{\circ} \mathrm{C}$ por $7 \mathrm{~h}$, o de carbono aumentou e o de oxigênio diminuiu a partir de $230^{\circ} \mathrm{C}$, por $5 \mathrm{~h}$ (Tabela 2).

\subsection{Análise colorimétrica}

Os valores da claridade e matriz amarelo (b*) foram maiores na testemunha, enquanto na matriz vermelho aumentou nos tratamentos a $170^{\circ} \mathrm{C}$ e diminuiu em temperaturas maiores (Tabela 3). 
Tabela 1 - Composição química da madeira termorretificada de Eucalyptus grandis. Table 1 - Chemical composition of heat treated Eucalyptus grandis wood.

\begin{tabular}{ccccccc}
\hline $\begin{array}{c}\text { Temperatura } \\
\left({ }^{\circ} \mathrm{C}\right)\end{array}$ & $\begin{array}{c}\text { Tempo } \\
(\mathrm{h})\end{array}$ & $\begin{array}{c}\text { Extrativos } \\
(\%)\end{array}$ & $\begin{array}{c}\text { Lignina } \\
\text { solúvel (\%) }\end{array}$ & $\begin{array}{c}\text { Lignina } \\
\text { insolúvel (\%) }\end{array}$ & $\begin{array}{c}\text { Lignina } \\
\text { total }(\%)\end{array}$ & $\begin{array}{c}\text { Holocelulose } \\
(\%)\end{array}$ \\
\hline Controle & Controle & $6,05 \mathrm{~b}$ & $3,39 \mathrm{a}$ & $25,36 \mathrm{a}$ & $28,76 \mathrm{a}$ & $65,19 \mathrm{~d}$ \\
\hline 170 & 3 & $5,43 \mathrm{a}$ & $3,47 \mathrm{a}$ & $25,83 \mathrm{a}$ & $29,30 \mathrm{a}$ & $65,26 \mathrm{~d}$ \\
& 5 & $5,06 \mathrm{a}$ & $3,52 \mathrm{a}$ & $26,43 \mathrm{a}$ & $29,96 \mathrm{a}$ & $64,98 \mathrm{~d}$ \\
& 7 & $4,72 \mathrm{a}$ & $3,53 \mathrm{a}$ & $25,47 \mathrm{a}$ & $29,01 \mathrm{a}$ & $66,27 \mathrm{~d}$ \\
\hline 200 & 3 & $5,92 \mathrm{~b}$ & $3,53 \mathrm{a}$ & $26,63 \mathrm{~b}$ & $30,36 \mathrm{~b}$ & $63,91 \mathrm{c}$ \\
& 5 & $6,78 \mathrm{c}$ & $3,31 \mathrm{a}$ & $27,68 \mathrm{~b}$ & $30,98 \mathrm{~b}$ & $62,23 \mathrm{c}$ \\
& 7 & $6,84 \mathrm{c}$ & $3,39 \mathrm{a}$ & $28,82 \mathrm{c}$ & $32,21 \mathrm{c}$ & $60,94 \mathrm{c}$ \\
\hline 230 & 3 & $8,15 \mathrm{~d}$ & $3,10 \mathrm{a}$ & $29,24 \mathrm{c}$ & $32,34 \mathrm{c}$ & $59,50 \mathrm{~b}$ \\
& 5 & $8,38 \mathrm{~d}$ & $3,57 \mathrm{a}$ & $32,90 \mathrm{~d}$ & $36,47 \mathrm{~b}$ & $55,13 \mathrm{a}$ \\
& 7 & $8,69 \mathrm{~d}$ & $3,31 \mathrm{a}$ & $33,86 \mathrm{~d}$ & $37,18 \mathrm{~b}$ & $54,12 \mathrm{a}$ \\
\hline
\end{tabular}

Médias seguidas de mesma letra na vertical não diferem pelo teste de Scott-Knott a 5\% de probabilidade.

Means followed by the same letter per column do not differ by Scott-Knott test at 5\% probability.

Tabela 2 - Composição elementar da madeira termorretificada de Eucalyptus grandis.

Table 2 - Elemental composition of heat treated Eucalyptus grandis wood.

\begin{tabular}{cccccc}
\hline Temperatura $\left({ }^{\circ} \mathrm{C}\right)$ & Tempo $(\mathrm{h})$ & Nitrogênio (\%) & Carbono (\%) & Hidrogênio (\%) & Oxigênio (\%) \\
\hline Controle & Controle & $0,683 \mathrm{a}$ & $47,30 \mathrm{a}$ & $5,79 \mathrm{~b}$ & $46,22 \mathrm{~b}$ \\
\hline \multirow{2}{*}{170} & 3 & $0,653 \mathrm{a}$ & $46,63 \mathrm{a}$ & $5,78 \mathrm{~b}$ & $46,92 \mathrm{~b}$ \\
& 5 & $0,683 \mathrm{a}$ & $46,19 \mathrm{a}$ & $5,68 \mathrm{~b}$ & $47,44 \mathrm{~b}$ \\
& 7 & $0,683 \mathrm{a}$ & $47,14 \mathrm{a}$ & $5,79 \mathrm{~b}$ & $46,38 \mathrm{~b}$ \\
\hline 200 & 3 & $0,730 \mathrm{a}$ & $46,86 \mathrm{a}$ & $5,61 \mathrm{~b}$ & $46,79 \mathrm{~b}$ \\
& 5 & $0,590 \mathrm{a}$ & $47,38 \mathrm{a}$ & $5,60 \mathrm{~b}$ & $46,42 \mathrm{~b}$ \\
& 7 & $0,680 \mathrm{a}$ & $47,71 \mathrm{a}$ & $5,46 \mathrm{a}$ & $46,14 \mathrm{~b}$ \\
\hline 230 & 3 & $0,806 \mathrm{a}$ & $47,96 \mathrm{a}$ & $5,36 \mathrm{a}$ & $45,86 \mathrm{~b}$ \\
& 5 & $0,710 \mathrm{a}$ & $49,40 \mathrm{~b}$ & $5,33 \mathrm{a}$ & $44,55 \mathrm{a}$ \\
& 7 & $0,776 \mathrm{a}$ & $49,24 \mathrm{~b}$ & $5,19 \mathrm{a}$ & $44,78 \mathrm{a}$ \\
\hline
\end{tabular}

Médias seguidas de mesma letra na vertical não diferem pelo teste de Scott-Knott a 5\% de probabilidade.

Means followed by the same letter per column do not differ by Scott-Knott test at 5\% probability.

Tabela 3 - Colorimetria da madeira termorretificada de Eucalyptus grandis.

Table 3 - Colorimetry changes in heat treated Eucalyptus grandis wood.

\begin{tabular}{ccccc}
\hline Temperatura $\left({ }^{\circ} \mathrm{C}\right)$ & Tempo $(\mathrm{h})$ & Claridade $(\mathrm{L})$ & Matriz vermelho $\left(\mathrm{a}^{*}\right)$ & Matriz amarelo $\left(\mathrm{b}^{*}\right)$ \\
\hline Controle & Controle & $70,14 \mathrm{f}$ & $12,04 \mathrm{c}$ & $23,59 \mathrm{f}$ \\
\hline \multirow{2}{*}{170} & 3 & $56,45 \mathrm{e}$ & $15,53 \mathrm{~d}$ & $21,03 \mathrm{e}$ \\
& 5 & $52,05 \mathrm{~d}$ & $15,03 \mathrm{~d}$ & $19,54 \mathrm{~d}$ \\
& 7 & $50,45 \mathrm{~d}$ & $14,53 \mathrm{~d}$ & $18,86 \mathrm{~d}$ \\
\hline 200 & 3 & $40,52 \mathrm{c}$ & $11,52 \mathrm{c}$ & $14,49 \mathrm{c}$ \\
& 5 & $37,80 \mathrm{~b}$ & $10,80 \mathrm{c}$ & $13,46 \mathrm{c}$ \\
& 7 & $34,58 \mathrm{a}$ & $8,94 \mathrm{~b}$ & $11,44 \mathrm{~b}$ \\
\hline 230 & 3 & $32,89 \mathrm{a}$ & $7,20 \mathrm{a}$ & $9,55 \mathrm{a}$ \\
& 5 & $31,64 \mathrm{a}$ & $6,32 \mathrm{a}$ & $9,52 \mathrm{a}$ \\
\hline
\end{tabular}

Médias seguidas de mesma letra na vertical não diferem pelo teste de Scott-Knott a 5\% de probabilidade Means followed by the same letter per column do not differ by Scott-Knott test at 5\% probability. 


\section{DISCUSSÃO}

\subsection{Análise química}

A redução dos extrativos a $170{ }^{\circ} \mathrm{C}$ se deve à volatilização dos extrativos polares, que são maioria nas folhosas e se decompõem em faixas de temperatura entre 130 e $250^{\circ} \mathrm{C}$ (MIRANDA; PEREIRA, 2002; MÉSZÁROS et al., 2007; MORAIS; PEREIRA, 2012).

A degradação das hemiceluloses concorda com relatos de decomposição desse componente a partir de $200{ }^{\circ} \mathrm{C}$ (MUSINGUZI et al., 2012; BARNETOA et al.; 2011), formando produtos solúveis em álcool/tolueno, o que aumenta o teor de extrativos totais (BRITO et al., 2008). Isso explica o aumento do teor de extrativos e a diminuição do teor de holoceluloses nas temperaturas de 200 e $230{ }^{\circ} \mathrm{C}$.

O teor de lignina solúvel constante e o aumento da lignina insolúvel e total a partir de $200^{\circ} \mathrm{C}$, se dá pela degradação dos outros constituintes, como os extrativos e hemiceluloses (ESTEVES; PEREIRA, 2009; SEVERO et al., 2012), mostrando que a ligninas são mais resistentes à degradação térmica (YILDIZ et al., 2006; TUMEN et al., 2010).

A temperatura mostrou-se mais efetiva na alteração da composição química da madeira que o tempo. Entretanto, houve diferença do tempo entre os tratamentos com a mesma temperatura para o teor de extrativos a $200{ }^{\circ} \mathrm{C}$, holoceluloses a $230{ }^{\circ} \mathrm{C}$ e lignina insolúvel e total a 200 e $230{ }^{\circ} \mathrm{C}$.

Outros autores encontraram resultados semelhantes, Severo et al. (2012) observaram alteração no teor de extrativos de 3,23 para 4,33\%, lignina de 29,18 para 30,40\% e holoceluloses de 67,6 para 65,27\% em Pinus elliottii termorretificado a $200{ }^{\circ} \mathrm{C}$ por $4 \mathrm{~h}$. Brito et al. (2008) encontraram alteração de 4,8 para $10,1 \%$ no teor de extrativos, 27,1 para 35,9\% no teor de lignina e de 67,9 para $62,17 \%$ no teor de holoceluloses para Eucalyptus grandis a $200{ }^{\circ} \mathrm{C}$.

\subsection{Análise elementar}

A diminuição no teor de holoceluloses com a temperatura concorda com o fato de esse componente ser o mais afetado pela termorretificação (YILDIZ et al., 2006; BRITO et al., 2008), por ter muitos grupos hidroxílicos, sua degradação diminui o teor de oxigênio. Enquanto a lignina, que é mais resistente ao processo de termorretificação, possui maior teor de carbono.

Extrativos polares, ricos em oxigênio, degradam em faixas de temperatura semelhantes às deste trabalho, enquanto os solúveis em diclorometano, ricos em carbono, possuem faixa de degradação de 250 a 550 ${ }^{\circ} \mathrm{C}$ (MÉSZÁROS et al., 2007).

\subsection{Análise colorimétrica}

A claridade reduziu de 70,14 para 31,64, mostrando perda de 54,89\%. A madeira nos tratamentos a $170{ }^{\circ} \mathrm{C}$ se tornou mais escura devido à volatilização dos extrativos polares (MÉSZÁROS et al., 2007; MOURA; BRITO, 2011). Entretanto, nas temperaturas de 200 e $230^{\circ} \mathrm{C}$, a redução da claridade ocorreu devido à degradação das holoceluloses (MOURA; BRITO, 2011). O coeficiente de correlação da claridade com o teor de extrativos e holoceluloses foi, respectivamente, de -0,702 e 0,687.

A matriz vermelho apresentou tendência inversa ao teor de extrativos totais, com aumento a $170^{\circ} \mathrm{C}$ e diminuição com temperaturas mais elevadas, apresentando coeficiente de correlação de -0,931. A matriz amarelo variou entre 23,59 e 8,52, com redução de $63,88 \%$. A coloração amarela é associada a cromóforos nos extrativos e na lignina (PINCELLI et al., 2012). A termorretificação transforma ou degrada esses compostos, o que reduz a matriz amarela.

A claridade a 170 e $200^{\circ} \mathrm{C}$, a matriz vermelho (a*) a $200^{\circ} \mathrm{C}$ e a matriz amarelo (b*) a 170 e $200^{\circ} \mathrm{C}$ variaram com o tempo entre tratamentos na mesma temperatura.

A claridade reduziu de 65,5 para 45,1 , a matriz vermelho (a*) de 12,8 para 10,5 e a matriz amarelo (b*) de 18,9 para 17,6 após a termorretificação de Eucalyptus saligna a $180{ }^{\circ} \mathrm{C}$ (PINCELLI et al., 2012). Para Eucalyptus grandis a $200^{\circ} \mathrm{C}$, a claridade caiu de 59,14 para 40,13 , a matriz vermelho (a*) de 18,51 para 9,76 e a matriz amarelo (b*) de 21,74 para 14,34 (MOURA; BRITO, 2011).

A mudança de cor não é desejada durante a secagem, mas o escurecimento pelo tratamento térmico pode agregar valor à madeira. Além disso, os métodos de coloração da madeira com tintas emitem tolueno e xileno, perigosos à saúde humana e ao meio ambiente (KORKUT et al., 2012). 


\section{CONCLUSÃO}

O tratamento térmico diminuiu o teor de extrativos nos tratamentos a $170{ }^{\circ} \mathrm{C}$ e aumentou a 200 e $230{ }^{\circ} \mathrm{C}$. Não alterou o teor de lignina solúvel, aumentou o teor de lignina insolúvel e total e diminuiu o teor de holoceluloses a partir de $200^{\circ} \mathrm{C}$. A análise elementar mostrou aumento do teor de carbono e diminuição do oxigênio após o tratamento a $230^{\circ} \mathrm{C}$ por 5 e $7 \mathrm{~h}$. A termorretificação reduziu a claridade, a matriz vermelho (a*) e a matriz amarelo (b*).

As alterações químicas devidas à termorretificação podem melhorar as propriedades físicas, e as alterações na cor ampliam suas possibilidades de mercado, fazendo que essa técnica apresente potencial de utilização para a madeira de eucalipto.

\section{AGRADECIMENTOS}

À Fundação de Amparo à Pesquisa do Estado de Minas Gerais (FAPEMIG), à Coordenação de Aperfeiçoamento de Pessoal de Nível Superior (CAPES) e ao Conselho Nacional de Desenvolvimento Científico e Tecnológico (CNPq), pelo apoio financeiro.

\section{REFERÊNCIAS}

ABRUZZI, R. C. et. al. Relação das propriedades mecânicas e densidade de postes de madeira de eucalipto com seu estado de deterioração.

Revista Árvore, v.36, n.6, p.1173-1181, 2012.

ALMEIDA, G.; BRITO, J.O.; PERRE, P. Changes in wood-water relationship due to heat treatment assessed on micro-samples of three Eucalyptus species. Holzforschung, v.63, n.1, p.80-88, 2009

\section{ASSOCIAÇÃO BRASILEIRA DE PRODUTORES} DE FLORESTAS PLANTADAS-ABRAF. Anuário estatístico da ABRAF: ano base 2011. Brasília: 2012. 145p.

BAL, B.C. e BEKTAS, I. The effects of heat treatment on physical properties of juvenile wood and mature wood of Eucalyptus grandis. Bioresources, v.7, n.4, p.5117-5127, 2012.

BARNETOA, A. G.; VILAB, C.; ARIZA, J. Eucalyptus kraft pulp production: Thermogravimetry monitoring. Thermochimica Acta, v.520, n.2, p.110-120, 2011.
BRITO, J. O. et al. Chemical composition changes in Eucalyptus and Pinus woods submitted to heat treatment. Bioresource Technology, v.99, n.18, p.8545-8548, 2008.

BROSSE, N. et al. Investigation of the chemical modifications of beech wood lignin during heat treatment. Polymer Degradation and Stability, v.95, n.9, p.1721-1726, 2010.

CADEMARTORI, P. H. G. et al. Modification of static bending strength properties of Eucalyptus grandis heat-treated wood. Materials Research, v.15, n.6, p.922-927, 2012.

CARNEIRO, M. E. et al. Classificação de lâminas de madeira de Pinus spp. contaminadas por fungos manchadores. Revista Árvore, v.3, n.2, p.369-375, 2013.

DUNDAR, T. et al. Effect of heat treament on the physical and mechanical properties of compression and opposite wood of black pine. Bioresources, v.7, n.4, p.5009-5018, 2012.

ESTEVES, B.; VIDEIRA, R.; PEREIRA, H. Chemistry and ecotoxicity of heat-treated pine wood extractives. Wood Science and Technology, v.45, n.4, p.661-676, 2011.

ESTEVES, B. et al. Heat-induced colour changes of pine (Pinus pinaster) and eucalypt (Eucalyptus globulus) wood. Wood Science and Technology, v.42, n.5, p.369-384, 2008.

ESTEVES, B.; PEREIRA, H. M. Wood modification by heat treatment: a review. Bioresources, v.4, n.1, p.370-404, 2009.

FURTADO, T. S. et al. Correlação entre teor de umidade e eficiência energética de resíduos de Pinus taeda em diferentes idades. Revista Árvore, v.36, n.3, p.577-582, 2012.

GARCIA, R. A. et al. Nondestructive evaluation of heat-treated Eucalyptus grandis Hill ex Maiden wood using stress wave method. Wood Science and Technology, v.46, n.1, p.41-52, 2012.

GOLDSCHIMID, O. Ultraviolet spectra. In: SARKANEN, K. V.; LUDWIG, C. H. Lignins: occurrence, formation, structure and reactions. New York: John Wiley \& Sons, 1971. p.241-298.

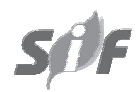

Revista Árvore, Viçosa-MG, v.38, n.4, p.765-770, 2014 
GOMIDE, J. L.; DEMUNER, B. J. Determinação do teor de lignina na madeira: método Klason modificado. O Papel, v.47, n.1, p.36-38, 1986

KOCAEFE, D. et al. Effect of heat treatment on the wettability of white ash and soft maple by water. Holz Roh-Werkst, v.66, n.5, p.355-361, 2008.

KORKUT, S. Performance of three thermally treated tropical wood species commonly used in Turkey. Industrial Crops and Products, v.36, n.1, p.355-362, 2012.

LIMA, C. M. et al. Comportamento da cor de lâminas de madeira de pau-marfim (Balfourodendron riedelianum) tratada com produtos de acabamento. Revista Árvore, v.37, n.2, p.377-384, 2013.

MÉSZÁROS, E.; JAKAB, E.; VÁRHEGYI, G. TG/ MS, Py-GC/MS and THM-GC/MS study of the composition and thermal behavior of extractive components of Robinia pseudoacacia. Journal of Analytical and Applied Pyrolysis, v.79, n.1, p.61-70, 2007.

MIRANDA, I.; PEREIRA, H. Variation of pulpwood quality with provenances and site in Eucalyptus globulus. Annals of Forest Science, v.59, n.3, p.283-291, 2002.

MORAIS, M. S. M.; PEREIRA, H. Variation of extractives content in heartwood and sapwood of Eucalyptus globulus trees. Wood Science and Technology, v.46, n.4, p.709-719, 2012.

MOURA, L. F.; BRITO, J. O. Efeito da termorretificação sobre as propriedades colorimétricas das madeiras de Eucalyptus grandis e Pinus caribaea var. hondurensis. Scientia Forestalis, v.39, n.89, p.69-76, 2011.

MUSINGUZi, W. B. et al. Thermal characterization of Uganda's Acacia hockii, Combretum molle, Eucalyptus grandis and Terminalia glaucescens for gasification. Biomass and Bioenergy, v.46, p.402-408, 2012.
PAULA, L. R. et al. Characterization of residues from plant biomass for use in energy generation. Revista Cerne, v.17, n.2, p.237246, 2011.

PINCELLI, A. L. P. S. M.; DE MOURA, L. M.; BRITO, J. O. Effect of thermal rectification on colors of Eucalyptus Saligna and Pinus Caribaea woods. Maderas. Ciencia y tecnología, v.14, n.2, p.239-248, 2012.

RATNASINGAM, J.; IORAS, F. Effect of heat treatment on the machining and other properties of rubberwood. European Journal of Wood Production, v.70, n.5, p.759-761, 2012.

SEVERO, E. T. D.; CALONEGO, F. W.; SANSÍGOLO, C. A. Physical and chemical changes in juvenile and mature woods of Pinus elliottii var. elliottii by thermal modification.

European Journal of Wood

Production, v.70, n.5, p.741-747, 2012.

SCHAFFER, E. L. Effect of pyrolytic temperatures on the longitudinal strenght of dry Douglas Fir. Journal of Testing and Evaluation, v.1, n.4, p.319-329, 1973.

SHI, J.; KOCAEFE, D.; ZHANG, J. Mechanical behaviour of Quebec wood species heat-treated using ThermoWood process. Holz RohWerkst, v.65, n.4, p.255-259, 2007.

THECHINICAL ASSOCIATION OF THE PULP AND PAPER INDUSTRY - TAPPI. Tappi Test Methods: 1992-1993. Atlanta: 1992.

TUMEN, I. et al. Changes in the chemical structure of thermally treated wood. Bioresources, v.5, n.3, p.1936-1944, 2010.

YILDIZ, S.; GEZER, E. D.; YILDIZ, Y. C. Mechanical and chemical behavior of spruce wood modified by heat. Building and Environment, v.41, n.12, p.1762-1766, 2006. 